\title{
Las competencias del docente de maestría en universidades colombianas: apreciaciones de alumnos y profesores ${ }^{1}$
}

\section{The competencies of the master's degree in Colombian universities: student and teacher evaluations}

Recibido: 18 de marzo de 2016 - Revisado: 29 de abril de 2016 - Aceptado: 13 de julio de 2016

Jhon Jairo Restrepo Aguirre ${ }^{2}$

Antonio Navío Gámez

\begin{abstract}
Resumen
Este artículo muestra los resultados de investigación sobre la visión competencial docente desde la óptica de sus protagonistas: enseñantes y aprendices. El diseño del estudio es descriptivo ex post facto desde la metodología empírico-analítica, compuesta por métodos cuantitativos, a partir de instrumentos como los cuestionarios y fuentes vinculadas con programas de maestría (docentes y alumnos). Su objetivo es analizar la apreciación de los informantes respecto al perfil docente actual y sus necesidades competenciales requeridas, todo desde la tarea docente e investigadora. Los resultados evidencian diferencias significativas que arrojan un conjunto de necesidades para la formación docente. Finalmente se exponen algunas reflexiones para una mejora de la formación de formadores en el contexto analizado.
\end{abstract}

\section{Palabras clave}

Educación superior, formación de maestría, competencias docentes.

\begin{abstract}
This article shows the results of research on the teaching competency view from the perspective of its protagonists: teachers and apprentices. The study design is descriptive $e x$ post facto from the empirical-analytical methodology, composed of quantitative methods, from instruments such as questionnaires and sources linked to master's programs (teachers and students). Its objective is to analyze the informants' appreciation of the current teaching profile and their required competency needs, all from the teaching and research task. The results prove significant differences that show a set of needs for teacher training. Finally, some reflections are presented for an improvement of the formation of trainers in the analyzed context.
\end{abstract}

\section{Keywords}

Higher education, master's degree, teaching competencies.

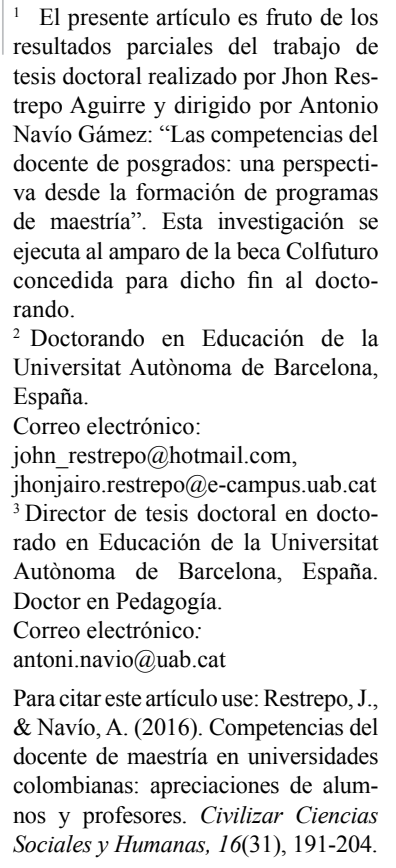

El presente artículo es fruto de los resultados parciales del trabajo de tesis doctoral realizado por Jhon Restrepo Aguirre y dirigido por Antonio Navío Gámez: "Las competencias del docente de posgrados: una perspectiva desde la formación de programas de maestría". Esta investigación se ejecuta al amparo de la beca Colfuturo concedida para dicho fin al doctorando.

${ }^{2}$ Doctorando en Educación de la Universitat Autònoma de Barcelona, España.

Correo electrónico:

john_restrepo@hotmail.com, jhonjairo.restrepo@e-campus.uab.cat

${ }^{3}$ Director de tesis doctoral en doctorado en Educación de la Universitat Autònoma de Barcelona, España.

Doctor en Pedagogía.

Correo electrónico:

antoni.navio@uab.cat

Para citar este artículo use: Restrepo, J., \& Navío, A. (2016). Competencias del docente de maestría en universidades colombianas: apreciaciones de alumnos y profesores. Civilizar Ciencias Sociales y Humanas, 16(31), 191-204. 


\section{Introducción}

Estudios doctorales destacan la importancia que tiene la formación de docentes universitarios en contextos europeos y latinoamericanos, revelando así escasez de estos estudios relacionados con la formación docente en programas de maestría (Navío, 2001; Mas, 2009; Pirela \& Prieto, 2006). En cuanto a las competencias y al rol del profesor universitario, teóricos analizan que las competencias no residen en las capacidades, sino en la movilización misma de una serie de recursos con que cuenta el docente y además en cómo involucra en su actuar una ética profesional aunada a estos recursos para así capitalizarlos a lo largo de la vida (Mas \& Tejada, 2013).

La capacidad productiva de un docente se define y evalúa atendiendo su rendimiento y actuación profesional en un entorno de educación, no solo de saberes, habilidades o destrezas ideales, sino la competencia integrada entre el saber, el saber hacer, el saber ser y el saber convivir (Toro, Ochoa, Villegas, \& Zea, 2004). Es decir, que una reflexión mesurada del quehacer del docente está estrechamente relacionada con el devenir y trasegar de la universidad misma, del papel que esta desempeña en su formación y en su apuesta al proyectar los futuros dirigentes y fuerza laboral de un país (Soto, 2019).

No cabe duda de que la cualificación docente de los profesores de maestría de las universidades colombianas es un tema importante por el hecho de existir una amplia y variada oferta de programas, y por la necesidad de regular este tipo de formación, tan primordial en el contexto educativo colombiano.

En ese sentido, la preocupación por la cualificación docente trasciende la mera capacitación cuando se adopta un modelo de competencias profesionales que, sobre la base de perfiles actuales y necesarios de acuerdo con estándares de calidad, permita saber cómo están valorados por parte de diferentes colectivos.
Dos ejes confluyen en este trabajo: los programas de maestría con su relevancia en la universidad colombiana y las competencias de unos docentes que, por el hecho de estar vinculados a maestría, no siempre tienen un único referente profesional, que es el de la docencia universitaria, sino que en muchas ocasiones son profesionales de otras áreas que se transforman, puntualmente, en maestros universitarios para volver, cuando finalizan la actividad, a sus tareas profesionales no universitarias.

\section{El formador de maestrías: campo de acción y competencias docentes}

El sistema educativo colombiano tiene fortalezas significativas en el contexto de la educación latinoamericana. La economía del país satisface las necesidades de talento humano calificado a través del sistema de educación superior, pero esto no sucede en proporción equitativa para toda la nación. Hay conciencia de que Colombia necesita una educación de calidad que garantice competitividad y que sea de acceso a toda la población.

Los programas de maestría en Colombia se rigen por las consideraciones del Ministerio de Educación Nacional y el Departamento Administrativo de Ciencia, Tecnología e Innovación (Colciencias), entes que reconocen la estrecha correspondencia entre ciencia, competitividad y desarrollo, aspectos que residen, esencialmente, en la formación del capital humano. Dicho capital humano en Colombia no es suficiente, razón por la cual la exigencia en torno a la calidad de los programas de maestría (maestrías de profundización y maestrías de investigación) permite el desarrollo de competencias en torno a la investigación, generación de nuevo conocimiento y aportes al desarrollo científico y tecnológico.

Desde esta perspectiva, la calidad de las instituciones de educación superior ha sido evaluada desde el servicio que prestan todos los sujetos que componen la estructura educativa. 
De acuerdo con Astin (1985) se identifican cinco niveles al definir calidad universitaria: calidad por valor agregado, calidad por contenidos, calidad por resultados, calidad por recursos disponibles y calidad por reputación. Aspectos que se enlazan con estas calidades, pero que atañen de modo directo a la labor del docente, son los que deben indagarse en los programas de maestría desde una lógica de competencias (Mas \& Tejada, 2013).

Pensar en las competencias desde la educación, es pensar en el desarrollo de una educación superior que se construye sobre la base de las competencias de estudiantes, docentes e investigadores. Es una de las demandas a la universidad del siglo XXI (García, Fraile, \& Sabán, 2008). Desde la óptica del profesor, se impone un análisis de perfil profesional, si nuestra intención es la de velar por el quehacer de este profesional.

Se requiere entonces, un análisis que enlace competencias profesionales, perfiles de referencia y necesidades de formación (Mas \& Tejada, 2013; Navío, 2006). Para poder investigar en torno a las competencias profesionales de los docentes de la educación superior, partimos de una concepción de competencia que contempla

[...] un conjunto de elementos combinados (conocimientos, habilidades, actitudes, etc.), que se integran atendiendo una serie de atributos personales (capacidades, motivos, rasgos de personalidad, aptitudes, etc.), tomando como referencia las experiencias personales y profesionales y que se manifiestan mediante determinados comportamientos o conductas en el contexto de trabajo (Navío, 2005, p. 71).

De acuerdo con Le Boterf (2008) asumimos que las competencias pueden entenderse desde los saberes movilizados, según unos recursos que se activan en función de las exigencias del contexto profesional y en consonancia con el criterio del mismo profesional (Mas \& Tejada, 2013).
Estudiar las competencias entonces, significa tener presente los siguientes aspectos: valorar los conocimientos, valorar las capacidades y otros atributos personales, considerar las experiencias, necesidades e intereses de las personas, tanto en lo personal como en lo profesional; valorar los comportamientos en el escenario laboral y estimar el propio contexto en el cual se desarrolla la competencia (Navío, 2005). Lo anterior admite distinguir tres fases, de acuerdo con la definición de competencia que se adopta en este estudio y que son los objetivos que lo delimitan:

- Identificar las competencias necesarias para realizar, con calidad, los roles y tareas demandados al docente de maestría.

- Interpretar las diferencias evidenciadas entre el perfil actual real del docente de maestría y las necesidades de formación requeridas para ejecutar la profesión docente con alta calidad.

- Definir criterios para abordar las necesidades de formación del docente de maestría.

Aclarado lo anterior, es preciso hablar sobre la tarea docente, tema central de este documento, entendiendo que con autonomía las universidades realizan o promueven cursos formativos que buscan contribuir a la mejora o adquisición de las competencias pedagógicas de los docentes; con la falencia de que estos intentos, en la mayoría de casos, no cuentan con la contundencia de calidad y seguimiento a posteriori de resultados, sin dejar de lado que en ciertos contextos la participación recae en la voluntad del mismo profesor.

Siguiendo los trabajos de Mas y Tejada (2013), Navío (2006), Valcárcel (2003) y Zabalza $(2003,2009)$-quienes presentan sus posturas respecto al perfil competencial del docente universitario-, nos adentramos en las competencias que se relacionan con la tarea docente, pero como dirían Mas y Ruiz (2007), competencias 
orientadas a satisfacer las demandas derivadas de la diversidad de los alumnos en el contexto actual y cómo no, según el paradigma de la exigencia de un formador competente en tanto conocimiento, comunicación, relaciones, didác- tica y gestión. En la tabla 1 se reportan las seis competencias definidas a partir del trabajo que se adelantó con grupos focales ${ }^{1}$, y sus 28 unidades competenciales relacionadas con la tarea docente.

Tabla 1

\section{Perfil competencial del docente de maestría para la tarea docente}

Competencias
Diseñar a través de equipos
interdisciplinarios los contenidos
programáticos según entorno,
perfil profesional y necesidades
institucionales y demandadas por
los educandos

Propender por la mejor conveniencia didáctica tanto personal como colectiva en la ejecución del binomio Enseñanza/ Aprendizaje

Orientar el proceso de aprendizaje integrando la tutoría como herramienta para el aprendizaje autónomo del estudiante

Ejecutar evaluación del proceso E/A con objetividad y atendiendo a la implementación de métodos y medios acordes con este propósito

Implicarse con efectividad en los procesos de mejora continua a la calidad educativa

Contribuir en la gestión administrativo- académica del escenario institucional que corresponda

\section{Elementos competenciales}

1. Determinar los grupos sujetos de aprendizaje, procurando armonizar la diversidad social

2. Definir y concretar los contenidos programáticos que formarán parte de los módulos didácticos

3. Proponer las metas a alcanzar en concordancia con el perfil competencial del formador, la filosofía institucional y la demanda social

4. Plantear metodología/s teniendo en cuenta el entorno diverso y el contexto de desarrollo académico

5. Elegir recursos didácticos en relación con las habilidades y estrategias pedagógicas del docente

6. Proyectar el sistema de evaluación del aprendizaje y los medios instrumentales para tal fin

7. Definir metodologías académicas conforme las metas propuestas

8. Dirigir la interacción didáctica y propender por una comunicación afable en la relación E/A

9. Emplear diversos recursos educativos en el proceso E/A

10. Gestionar los recursos e infraestructura aportados por la institución

11. Planear fases de tutorización, en consecuencia con las metas de la asignatura y la diversidad de los educandos

12. Dirigir el proceso de apropiación de conocimiento del estudiante facilitando los medios necesarios

13. Gestar relaciones interpersonales óptimas para una comunicación cordial y amable con el alumno

14. Gestionar las nuevas TIC para beneficiar el binomio E/A en entornos virtuales

15. Implicar uso de los métodos e instrumentos que contribuyen a la auto, hetero y coevaluación educativa

16. Demostrar ética respecto a la evaluación educativa

17. Aplicar el modelo evaluativo institucional de acuerdo con el plan curricular diseñado

18. Realizar evaluación integral a los diferentes elementos que componen el proceso E/A

19. Verificar el logro de aprendizajes de los educandos

20. Tomar decisiones basándose en la información obtenida

21. Proyectar necesidades de formación que contribuyan a la mejora del proceso E/A

22. Interrelacionarse con el ámbito profesional y social para actualizarse continuamente

23. Construir a través de equipos interdisciplinarios recursos pedagógicos que ayuden a las actuales necesidades de formación

24. Cooperar en las tareas de adaptación de programas que comprendan acciones de mejoramiento al proceso $\mathrm{E} / \mathrm{A}$

25. Promocionar la realización y asistencia a eventos académicos que conlleven mejora del proceso $\mathrm{E} / \mathrm{A}$

26. Vincularse a equipos de trabajo para programar los currículos según área de conocimiento

27. Involucrarse activamente en las jornadas de actualización e innovación docente

28. Participar en la evaluación a la gestión administrativa

Fuente: elaboración propia. 
En cuanto a los resultados de esta investigación desde la visión de los protagonistas, se destaca la necesidad de formar a los docentes de maestría en aspectos que atañen directamente a la enseñanza y el aprendizaje en coherencia con el contexto en que se desempeñan, para así atender las necesidades sociales y educativas, $\mathrm{y}$ ofrecer educación de alta calidad y competencial a los futuros magísteres, lo que se traduce en mayores oportunidades laborales. Lo anterior también implica tener en cuenta la postura del docente, del alumno y de los directivos en una triangulación orientada desde las necesidades de formación para la excelencia.

\section{Problema de investigación y método}

Esta investigación se contextualiza en el campo de las necesidades de formación de los docentes de programas de maestría en Colombia, analizadas desde la enseñanza y el aprendizaje, donde los profesores y estudiantes de maestría, además de las instituciones, son quienes se encargan de gestar nuevas y mejores prácticas de formación docente, alrededor de los procesos formativos y de proyección social que permita un alto desempeño competencial tanto en docencia como en investigación.

\section{Objetivo.}

Analizar las apreciaciones de dominio y necesidad docente e investigativa de los profesores, respecto a las competencias (diseño, didáctica, orientación, evaluación, mejora continua y gestión) definidas en el estudio para la formación de docentes desde la visión del mismo educador y los alumnos.

\section{Enfoque metodológico.}

El presente estudio es descriptivo, ex post facto desde la metodología empírico-analítica (Bisquerra, 2012). Además combina el estudio de caso atendiendo lo manifestado por Latorre, Del Rincón y Arnal (2005) y entendiendo que el estudio de caso corresponde a la forma más adecuada para hacer investigación en el proceso de comprender aspectos exclusivos o característicos de un tema por estudiar, desarrollado desde un enfoque cualitativo, debiendo orientarse como una táctica dirigida a la toma de decisiones. Es decir, se pretende evidenciar un estado actual a partir de las perspectivas de los informantes, docentes y alumnos de programas de maestría.

\section{Población y muestra.}

La población objeto de estudio está compuesta por el grupo de directivos adscritos a la gestión, los maestros de tres universidades privadas colombianas ${ }^{2}$-vinculados a la formación en programas de maestría- y los alumnos de la última cohorte en desarrollo de los programas de maestría que se asocian con el estudio ${ }^{3}$.

\section{Gráfica 1.}

Población universidades privadas colombianas
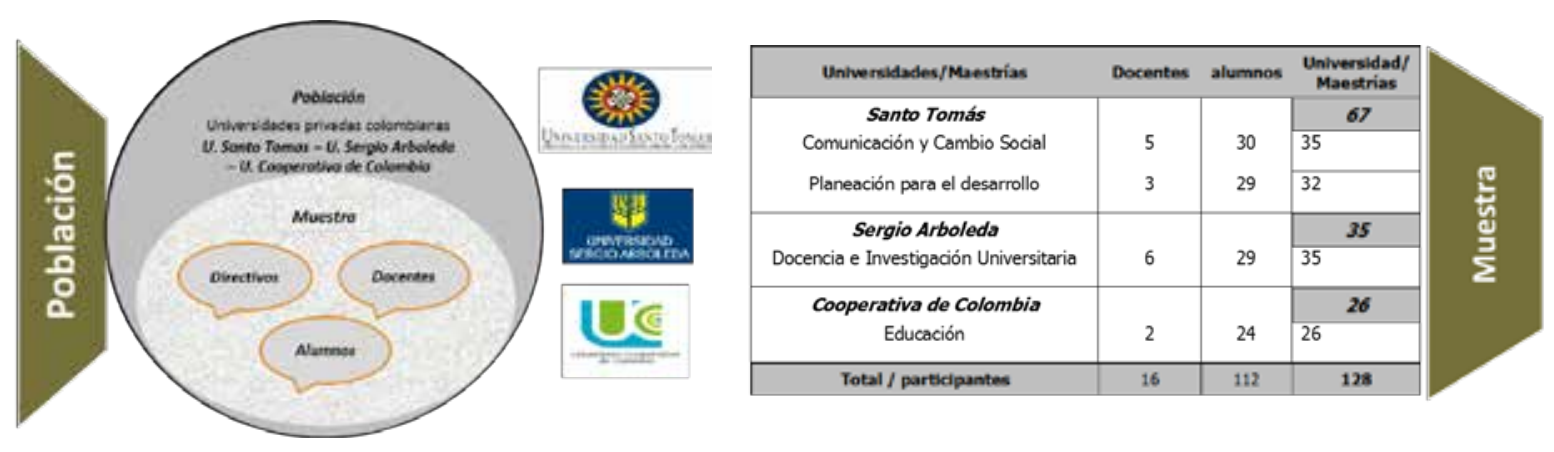

Fuente: elaboración propia. 
La muestra se corresponde con un patrón de muestreo no probabilístico compuesto por 112 estudiantes pertenecientes a última cohorte de cuatro programas de maestrías en tres universidades colombianas y 16 docentes $\mathrm{y}$ directivos de dichos programas, quienes respondieron a un cuestionario que tenía unas variables previas de caracterización de los informantes más un listado competencial, en el que los encuestados debían valorar el dominio global de las competencias por parte de los docentes, al tiempo que el grado de necesidad de presencia de cada una de las competencias.

\section{Instrumentos.}

Los instrumentos se diseñan y elaboran a partir del proceso de discusión y validación de la propuesta competencial trabajada con focus group $^{4}$ y validada por jueces expertos ${ }^{5}$. Es así como se obtiene la estructura definida para el cuestionario, atiende modelos competenciales mixtos de investigadores que han trabajado en delimitación del perfil competencial del docente universitario a partir de la función docente e investigadora y su relación con la generación de conocimiento científico sobre esta temática (Mas, 2009; Navío, 2001; Pirela \& Prieto, 2006).

Para los docentes $^{6}$ y estudiantes ${ }^{7}$ de maestría se diseñó un cuestionario compuesto por un listado competencial de 28 elementos para el ejercicio docente y 22 elementos para las competencias investigadoras, las cuales se valoraron en una escala de Likert de cinco niveles de respuesta, donde 1 es bajo y 5 es alto, así mismo se incluyó un componente de 25 capacidades y valores que se evaluaron en dos escenarios, el primero: cuenta actualmente (sí, no) y el segundo: necesidad para excelencia (necesidad nula, necesidad mediana y alta necesidad) igualmente en escala de Likert.

Así mismo, se trianguló la información de docentes y alumnos, para contrastar los datos obtenidos en la búsqueda de las necesidades de formación, así como los perfiles por competencias del formador de maestrías, desde la autoevaluación, heteroevaluación y coevaluación, sin dejar de lado el entorno en que se desempeña, el cual cuenta con características indispensables según esboza Fernández, Tejada, Jurado de los Santos, Navío y Ruiz (2000).

\section{Análisis de la información.}

El análisis de las aportaciones se hizo con el programa estadístico SPSS, versión 22.0. Además de un análisis descriptivo e inferencial entre el dominio actual y la necesidad valorada por los docentes y alumnos, haciendo una comparación de puntuaciones medias con el fin de comprobar si las diferencias entre dominio y necesidad mostraban niveles de significatividad. Sobre la base de esas diferencias y de la significación de las mismas, fue como se procedió a interpretar los resultados.

Por último y por la concreción que requiere este artículo, solo se trabaja con los datos obtenidos de las fuentes primarias ${ }^{8}$, sobre las seis competencias docentes (diseño, ejecución, orientación, evaluación, mejora continua y gestión) y los datos resultantes de las 28 tareas competenciales que las componen; pero no se presentan los datos resultantes de las cuatro competencias investigadoras y sus 22 tareas tratadas en el estudio. Tampoco se incluye lo correspondiente a los datos derivados de las entrevistas en profundidad hechas a expertos de los programas implicados en la muestra. Estos quedan pendientes de análisis en otros contextos académicos.

\section{Presentación de los resultados de la investigación}

\section{Caracterización de la muestra.}

En la tabla 2 se observa un resumen de la caracterización de la muestra tanto de docentes como de alumnos implicados en la investigación. 
Tabla 2

Caracterización de la muestra

\begin{tabular}{|c|c|c|}
\hline Variables & Docentes & Alumnos \\
\hline Edad & 46,31 años & 35,76 años \\
\hline Género & $\begin{array}{l}\text { Femenino: } 43,8 \% \\
\text { Masculino: } 56,3 \%\end{array}$ & $\begin{array}{l}\text { Femenino: } 60,7 \% \\
\text { Masculino: } 39,3 \%\end{array}$ \\
\hline Titulación máxima & $\begin{array}{c}\text { Doctorado: } 43,8 \% \\
\text { Maestría: } 56,2 \%\end{array}$ & $\begin{array}{c}\text { Maestría: } 4,5 \% \\
\text { Especialización: } 71,4 \% \\
\text { Pregrado: } 24,1 \%\end{array}$ \\
\hline Formación continua pedagogía & $\begin{array}{l}\text { Con actualización: } 50 \% \\
\text { Sin actualización: } 50 \%\end{array}$ & \\
\hline Experiencia docente & Posgrados: $100 \%$ & $\begin{array}{c}\text { Posgrados: } 5,4 \% \\
\text { Pregrados: } 35,7 \% \\
\text { Fuera universidad: } 46,4 \% \\
\text { Sin experiencia: } 12,5 \%\end{array}$ \\
\hline Experiencia formación formadores & $\begin{array}{c}\text { Docentes universitarios: } 81,3 \% \\
\text { Docentes no universitarios: } 56,3 \%\end{array}$ & $\begin{array}{c}\text { Docentes universitarios: } 14,3 \% \\
\text { Docentes no universitarios: } 19,6 \%\end{array}$ \\
\hline Práctica en investigación & $\begin{array}{l}\text { Grupo: } 87,5 \% \\
\text { Individual: } 50 \%\end{array}$ & $\begin{array}{c}\text { Grupo: } 32,1 \% \\
\text { Individual: } 23,2 \%\end{array}$ \\
\hline Experiencia gestión administrativa & $\begin{array}{c}\text { Gestión universitaria: } 75 \% \\
\text { Gestión no universitaria: } 25 \%\end{array}$ & $\begin{array}{l}\text { Gestión universitaria: } 31,3 \% \\
\text { Gestión no universitaria: } 36,6 \%\end{array}$ \\
\hline Universidades en que trabajan & $\begin{array}{l}\text { USA: } 37 \% \\
\text { USTA: } 50 \% \\
\text { UCC: } 13 \%\end{array}$ & \\
\hline Maestrías que imparten/cursan & $\begin{array}{c}\mathrm{DIU}^{9}: 37,5 \% \\
\mathrm{CCS}^{10}: 31,3 \% \\
\text { PD }^{11}: 18,8 \% \\
\mathrm{E}^{12}: 12,5 \%\end{array}$ & $\begin{array}{c}\text { DIU: } 25,9 \% \\
\text { CCS: } 26,8 \% \\
\text { PD: } 25,9 \% \\
\text { E: } 21,4 \%\end{array}$ \\
\hline Antigüedad institución & $\begin{array}{c}\text { Docente novel }(<5 \text { años): } 62,5 \% \\
\text { Docente experto: } 37,5 \%\end{array}$ & \\
\hline Ámbitos de conocimiento & $\begin{array}{c}\text { Ciencias Soc. Hum.: 68,8\% } \\
\text { Ciencias Educac.: } 31,2 \%\end{array}$ & \\
\hline Vinculación contractual & $\begin{array}{c}\text { Todas las categorías: } 50 \% \\
\text { Catedráticos: } 25 \% \\
\text { Investigadores: } 19 \% \\
\text { Administrativos: } 6 \%\end{array}$ & \\
\hline Dedicación & $\begin{array}{l}\text { Tiempo completo: } 75 \% \\
\text { Tiempo parcial: } 25 \%\end{array}$ & \\
\hline
\end{tabular}

Fuente: elaboración propia.

\section{Valoración del dominio y las nece- sidades competenciales del docente.}

En la tabla 3 se muestran las valoraciones de los estudiantes y profesores sobre el dominio

actual y las necesidades competenciales de los docentes. 
Tabla 3

Valoración de las competencias docentes por parte de alumnos y profesores

\begin{tabular}{|c|c|c|c|c|c|c|}
\hline \multirow[b]{2}{*}{ Competencia 1: Diseño } & \multicolumn{3}{|c|}{ Alumnos } & \multicolumn{3}{|c|}{ Docentes } \\
\hline & $\mathbf{D A}^{13}$ & $\mathbf{N E}^{14}$ & Signif. & DA & NE & Signif. \\
\hline 1. Determina los grupos de aprendizaje & 3,8 & 4,3 & $\mathrm{P}=0,000$ & 3,1 & 4,0 & $\mathrm{P}=0,008$ \\
\hline 2. Define contenidos programáticos & 4,2 & 4,4 & $\mathrm{P}=0,029$ & 4,1 & 4,7 & \\
\hline 3. Propone las metas a alcanzar & 4,1 & 4,4 & $\mathrm{P}=0,007$ & 3,6 & 4,2 & $\mathrm{P}=0,039$ \\
\hline 4. Plantea metodologías & 3,8 & 4,5 & $\mathrm{P}=0,000$ & 4,1 & 4,7 & $P=0,033$ \\
\hline 5. Elige recursos didácticos & 3,7 & 4,4 & $\mathrm{P}=0,000$ & 3,8 & 4,5 & $\mathrm{P}=0,002$ \\
\hline 6. Proyecta el sistema de evaluación & 3,9 & 4,3 & $\mathrm{P}=0,000$ & 3,7 & 4,5 & $\mathrm{P}=0,008$ \\
\hline \multicolumn{7}{|l|}{ Competencia 2: Ejecución } \\
\hline 7. Define metodologías acordes con las metas & 3,9 & 4,4 & $\mathrm{P}=0,000$ & 4,1 & 4,5 & \\
\hline 8. Dirige la interacción didáctica & 3,9 & 4,4 & $\mathrm{P}=0,000$ & 4,3 & 4,5 & \\
\hline 9. Emplea diversos recursos educativos & 3,6 & 4,4 & $P=0,000$ & 4 & 4,5 & $P=0,029$ \\
\hline 10. Gestiona los recursos e infraestructuras & 3,6 & 4,4 & $\mathrm{P}=0,000$ & 3,5 & 4,4 & $\mathrm{P}=0,005$ \\
\hline \multicolumn{7}{|l|}{ Competencia 3: Orientación } \\
\hline 11. Planea fases de tutorización & 3,4 & 4,2 & $P=0,000$ & 3,8 & 4,4 & \\
\hline 12. Dirige el proceso de apropiación & 3,7 & 4,3 & $\mathrm{P}=0,000$ & 3,8 & 4,5 & $P=0,046$ \\
\hline 13. Gestiona relaciones interpersonales & 4,3 & 4,3 & & 4,5 & 4,5 & \\
\hline 14. Gestiona TIC & 3,6 & 4,4 & $P=0,000$ & 3,4 & 4,6 & $P=0,006$ \\
\hline \multicolumn{7}{|l|}{ Competencia 4: Evaluación } \\
\hline 15. Uso de métodos e instrumentos & 3,7 & 4,4 & $\mathrm{P}=0,000$ & 4 & 4,7 & $\mathrm{P}=0,008$ \\
\hline 16. Comportamiento ético respecto a evaluación & 4,3 & 4,4 & & 4,6 & 4,7 & \\
\hline 17. Aplica modelo evaluativo institucional & 4,2 & 4,4 & $\mathrm{P}=0,031$ & 3,7 & 4,4 & $\mathrm{P}=0,022$ \\
\hline 18. Realiza evaluación integral & 3,9 & 4,4 & $\mathrm{P}=0,003$ & 3,8 & 4,6 & $\mathrm{P}=0,019$ \\
\hline 19. Verifica el logro de aprendizajes & 3,8 & 4,4 & $\mathrm{P}=0,000$ & 3,8 & 4,5 & $\mathrm{P}=0,030$ \\
\hline 20. Toma decisiones derivadas de la evaluación & 3,9 & 4,3 & $\mathrm{P}=0,018$ & 3,9 & 4,6 & $\mathrm{P}=0,031$ \\
\hline \multicolumn{7}{|l|}{ Competencia 5: Mejora continua } \\
\hline 21. Proyecta mejora del sistema educativo & 3,7 & 4,3 & $\mathrm{P}=0,000$ & 3,4 & 4,5 & $\mathrm{P}=0,004$ \\
\hline 22. Se interrelaciona para actualizarse & 4 & 4,2 & & 3,8 & 4,3 & \\
\hline 23. Construye recursos pedagógicos & 3,6 & 4,3 & $\mathrm{P}=0,000$ & 3,8 & 4,5 & $\mathrm{P}=0,015$ \\
\hline 24. Coopera adaptando programas & 3,8 & 4,3 & $\mathrm{P}=0,000$ & 3,8 & 4,5 & $\mathrm{P}=0,008$ \\
\hline \multicolumn{7}{|l|}{ Competencia 6: Gestión institucional } \\
\hline 25. Promociona eventos académicos & 3,7 & 4,3 & $\mathrm{P}=0,000$ & 3,8 & 4,7 & $P=0,008$ \\
\hline 26. Se vincula a equipos de trabajo & 3,6 & 4,4 & $\mathrm{P}=0,000$ & 3,3 & 4,6 & $\mathrm{P}=0,003$ \\
\hline 27. Se involucra en actualización & 3,7 & 4,3 & $\mathrm{P}=0,001$ & 4,1 & 4,4 & \\
\hline 28. Participa en la evaluación de la gestión & 3,7 & 4,4 & $\mathrm{P}=0,000$ & 3,5 & 4,3 & \\
\hline
\end{tabular}

Fuente: elaboración propia.

En la gráfica 2 se evidencia necesidad competencial en los docentes para alcanzar el nivel de excelencia (línea naranja), desde la perspectiva de los alumnos y los mismos maestros. Este hecho lo podemos constatar si reparamos en las gráficas 2 y 3 . Por ejemplo en la competencia 1 "diseño", el elemento competencial 1 "Determina los grupos de aprendizaje" para los estudiantes la media en DA es 3,8 y en NE es 4,3 para una diferencia significativa de 0,000 (véase tabla 3 ). 


\section{Gráfica 2}

Valoración de las competencias docentes por parte de los alumnos

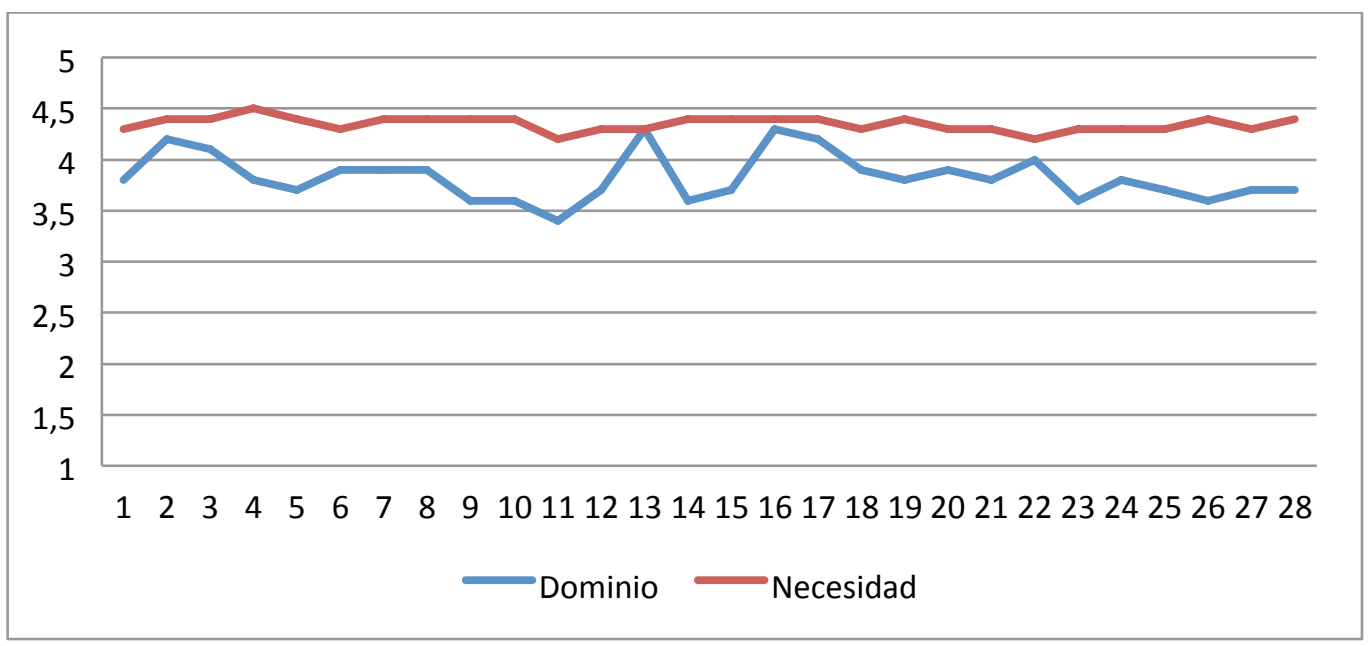

Fuente: elaboración propia.

Gráfica 3

Valoración de las competencias docentes por parte de los profesores

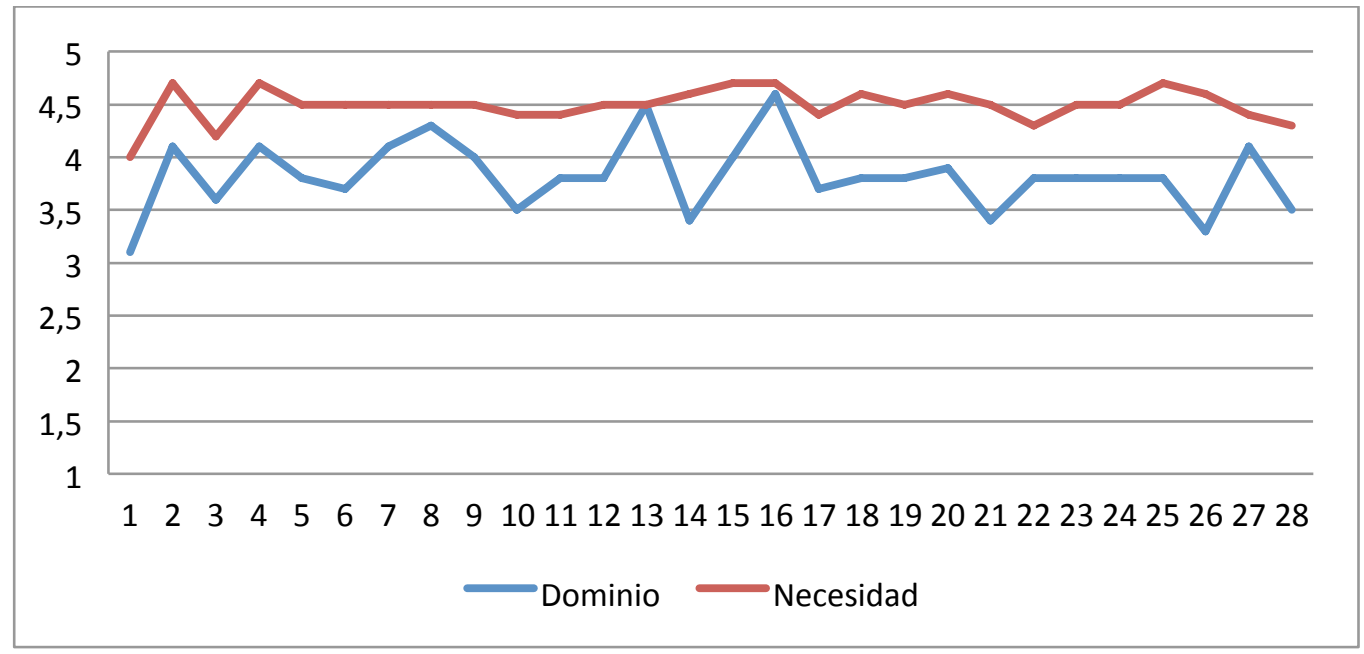

Fuente: elaboración propia.

Al realizar una valoración de las competencias docentes por parte de los alumnos y profesores podemos evidenciar que:

- La competencia de diseño, aunque presenta necesidades en todos los aspectos, constata que el aspecto menos prioritario en cuanto a necesidad es el que se asocia con los contenidos de formación. Se infiere que esto debe obedecer a la experticia del docente de maestría en los contenidos de la formación.

- En la competencia de ejecución, todos los aspectos manifiestan necesidad. Sin duda, las habilidades didácticas de los docentes de maestría parecen ser una necesidad muy clara y en lo 
que parece estar de acuerdo los informantes.

- En la competencia de orientación, tan solo un elemento no reporta necesidades según los estudiantes, se trata de la gestión de las relaciones interpersonales. En tanto que al sentir del docente la necesidad se ubica en la gestión de las TIC y la dirección del proceso de apropiación de conocimiento.

- En la competencia de evaluación para los participantes no se presenta necesidad en los aspectos éticos vinculados a la evaluación. Por su parte, el docente evidencia necesidad en un mayor grado en el uso de métodos e instrumentos de evaluación seguido de la evaluación integral; la aplicación del modelo evaluativo institucional, así como la verificación al logro del aprendizaje y la toma de decisiones derivadas de la evaluación.

- La competencia de mejora continua, de acuerdo con los docentes, refleja un aspecto que no presenta necesidad, asociado con la forma de interrelacionarse y actualizarse, situación coherente con el contexto de desempeño. Para los alumnos, no hay necesidad de sus profesores en lo que respecta a la actualización.

- La competencia de gestión institucional es una de las asignaturas pendientes de estos docentes. La poca vinculación con la institución universitaria, en la mayoría de los casos, hace que los estudiantes perciban que están necesitados de desarrollar esta competencia. Por su parte, los profesores demandan vinculación a equipos de trabajo y promoción de eventos académicos.

\section{Conclusiones}

Hay, desde un punto de vista estadístico, menos necesidad en las valoraciones de los do- centes que en las valoraciones de los alumnos. Esto no indica que los docentes no sean críticos. Al contrario, sopesan su análisis en lo que verdaderamente consideran necesario. Un recorrido por cada bloque competencial, nos permite una conclusión parcial que, en cualquier caso, deberá matizarse y enriquecerse con los aportes de las entrevistas.

En la competencia de diseño, hay coincidencia en su alta necesidad tanto para docentes como para alumnos, excepto una discrepancia en cuanto a los contenidos, esto debido al dominio del contenido por parte de los docentes en su condición de expertos. Igualmente, se infiere que es importante que en el diseño del contenido, el docente sea lo suficientemente explícito para que el alumno lo perciba claramente.

En la competencia de ejecución, se encuentra acuerdo en lo correspondiente a recursos y gestión de infraestructuras; es decir, que lo que depende del docente no evidencia necesidad pero lo que depende del contexto sí. Con lo cual, más que una necesidad docente, existe una necesidad institucional, vinculada a la disponibilidad de recursos e infraestructuras para la docencia.

En la competencia de orientación, aunque los maestros consideran que realizan una planificación de la tutoría, los alumnos reconocen que esto debería mejorarse. Por otro lado, se requiere atención y mejora del proceso de apropiación y gestión de las TIC, ello puede deberse, por una parte a que algunos docentes no pertenecen a la generación digital; por tal razón, su manejo de las nuevas TIC es lento y complejo y por otra parte, a que se requieren planes de tutorización pertinentes con la necesidad.

En la competencia de evaluación, hay un acuerdo entre los informantes respecto a la necesidad. En lo ético, nos hallamos ante una evaluación muy cercana al estudiante, esto por la evidencia de programas específicos, de grupos reducidos y con gran cuidado institucional. 
En la competencia de mejora continua, se evidencia desde los informantes que el docente está más cercano a la realidad y se interrelaciona para actualizarse, pues son profesores de maestría expertos en el campo en que desarrollan su tarea.

En la competencia de gestión institucional, la discrepancia con los alumnos se gesta en la actualización docente y la evaluación de la gestión, entendiendo con esto nuevamente un llamado a los maestros para hacerse más partícipes del entorno universitario.

Por último y de acuerdo con Restrepo (2008), se debe atender la mejora de los dos pilares fundamentales de la educación: el currículo y la evaluación, con lo cual, y según García y Castro (2012) se entiende la actualización y formación permanente de los docentes como un criterio de relevancia para definir la calidad educativa. Lo anterior conlleva requerir un cambio en la función docente desde la demanda de una formación permanente soportada en competencias que igualmente contribuyan al desarrollo competencial de sus alumnos.

También se evidencia la necesidad de atender a la voz directa de los implicados para matizar las necesidades. Esta voz, que proviene de las entrevistas, permitirá ajustar las necesidades de forma más clara, al tiempo que contextualizará para cada situación institucional, las posibles actuaciones de formación docente.

\section{Notas}

${ }^{1}$ Se realizaron cuatro grupos focales con cada uno de los programas de maestría participantes en el estudio en los cuales se vincularon 26 expertos.

${ }^{2}$ Universidad Sergio Arboleda (USA); Universidad Cooperativa de Colombia (UCC) y Universidad Santo Tomás (USTA).

${ }^{3}$ Maestría en Docencia e Investigación Universitaria (USA); maestría en Educación (UCC) y las maestrías en: Planeación para el Desarrollo y Comunicación y Cambio Social (USTA).

${ }^{4}$ Se realizaron ocho grupos de discusión con la participación de 26 directivos de las maestrías participantes en Colombia.

${ }^{5}$ Los jueces expertos que validaron la propuesta de cuestionarios pertenecen a la Universidad Autónoma de Barcelona (UAB).

6 Cuestionario compuesto por cuatro variables: personales, formativas, sociolaborales y generales del programa.

${ }^{7}$ Cuestionario compuesto por tres variables: personales, formativas y generales del programa.

${ }^{8}$ Las fuentes primarias de información en esta investigación son: docentes y alumnos que imparten y realizan programas de maestría en las universidades colaboradoras en el estudio.

${ }^{9}$ Maestría en Docencia e Investigación Universitaria (Universidad Sergio Arboleda).

${ }^{10}$ Maestría en Comunicación y Cambio Social (Universidad Santo Tomás).

11 Maestría en Planeación para el Desarrollo (Universidad Santo Tomás).

12 Maestría en Educación (Universidad Cooperativa de Colombia).

${ }^{13}$ Dominio actual.

${ }^{14}$ Necesidad para la excelencia.

\section{Referencias}

Astin, A. W. (1985). Achieving Educational Excellence. San Francisco: Jossey-Bass.

Bisquerra, R. (Coord.). (2012). Metodología de la investigación educativa ( $3^{\mathrm{a}}$ ed.). Madrid: La Muralla. 
Ferrandez, A., Tejada, J., Jurado de los Santos, P., Navío, A., \& Ruiz, C. (2000). El formador de formación profesional $y$ ocupacional. Barcelona: Octaedro.

García-Fraile, J. A., \& Sabán, C. (Coords.) (2008). Un nuevo modelo de formación para el siglo XXI: la enseñanza basada en competencias. Barcelona: DaVinci.

García, R., \& Castro. Z, A. (2012). La formación permanente del profesorado basada en competencias. Estudio exploratorio de la percepción del profesorado de Educación Infantil y Primaria. Educatio Siglo XXI, 30(1), 297-322. Recuperado de http://revistas.um.es/educatio/article/view/149251

Latorre, A., Del Rincón, D., \& Arnal, J. (2005). Bases metodológicas de la investigación educativa. Barcelona: Ediciones Experiencia.

Le Boterf, G. (2008). Repenser la compétence. Paris: Eyrolles.

Mas, Ó. (2009). El perfil competencial del profesorado universitario en el Espacio Europeo de Educación Superior (Tesis doctoral). Universidad Autónoma de Barcelona, Bellaterra, España.

Mas, Ó., \& Ruiz, C. (mayo, 2007). Competencias profesionales del profesor universitario desde una visión integradoras de sus funciones y perfiles. Comunicación del III congreso internacional de formación del profesorado. Innovación, formación y profesionalización educativa, Universidad de Granada, España.

Mas, Ó., \& Tejada, J. (2013). Funciones y competencias en la docencia universitaria. Madrid: Síntesis.

Navío, A. (2001). Las competencias del formador de formación continua. Análisis desde los programas de formación de formadores (Tesis doctoral). Universidad Autónoma de Barcelona, Bellaterra, España.

Navío, A. (2005). Las competencias profesionales del formador. Una visión desde la formación continua. Barcelona: OctaedroEUB.

Navío, A (2006). Análisis y detección de necesidades. En J. Tejada \& V. Giménez (Coords.), Formación de formadores. Escenario Aula (Tomo 1, pp. 71-152). Madrid: Thomson.

Pirela, L., \& Prieto, L. (2006). Perfil de competencias del docente en la función de investigador y su relación con la producción intelectual. Opción, 22 (50), 159-177. Recuperado de http://www. produccioncientifica.luz.edu.ve/index. php/opcion/article/view/6383/6371

Restrepo, J. J. (2008). Diagnóstico sobre el estado de la práctica evaluativa: retroalimentación constante al alumno, realizada por docentes/tutores de programas virtuales, en la Universidad Colegio Mayor de Cundinamarca. Cuadernos de la Maestría en Docencia e Investigación Universitaria, 1, 127142. Recuperado de http://190.85.246.40/ docencia/cuadernosMaestria.pdf

Soto, D. (2009). El profesor universitario de AméricaLatina:Haciaunaresponsabilidad ética, científica y social. Revista Historia de la Educación Latinoamericana, 13, 166-168. Recuperado de http://www. redalyc.org/articulo.oa? $\mathrm{id}=86912384010$

Toro, P., Ochoa, P., Villegas, G., \& Zea, C. (octubre, 2004). Competencias deseables de un docente universitario en el uso de las tecnologías de información y comunicación (TIC). Ponencia presentada en el 
"I Congreso Internacional de Educación mediada por Tecnología", Barranquilla, Colombia. Recuperado de: http://www. colombiaaprende.edu.co/html/mediateca/1607/articles-74000_archivo.pdf

Valcárcel, M. (Coord.). (2003). La preparación del profesorado universitario español para la Convergencia europea en Educación Superior. Recuperado de http:// campus.usal.es/ ofeees/ESTUDIOS_INFORMES_GRALES/informe_final.pdf
Zabalza, M. (2003). Competencias docentes del profesorado universitario: calidad y desarrollo profesional. Madrid: Narcea.

Zabalza, M (2009). Ser profesor universitario hoy. La cuestión universitaria, 5, 6981 Recuperado de http://tecnologiaedu. us.es $/ \mathrm{mec} 2011 / \mathrm{htm} / \mathrm{mas} / 3 / 31 / 47 . \mathrm{pdf}$ 
